Supplement of The Cryosphere, 14, 4103-4120, 2020

https://doi.org/10.5194/tc-14-4103-2020-supplement

(C) Author(s) 2020. This work is distributed under

the Creative Commons Attribution 4.0 License.

(c) (i)

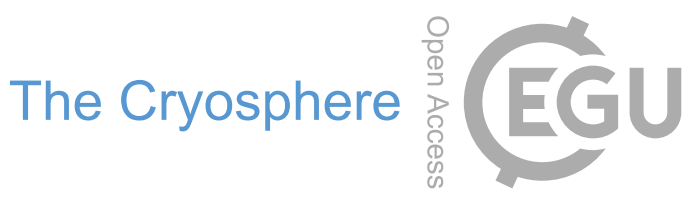

Supplement of

\title{
Distribution and seasonal evolution of supraglacial lakes on Shackleton Ice Shelf, East Antarctica
}

Jennifer F. Arthur et al.

Correspondence to: Jennifer F. Arthur (jennifer.arthur@durham.ac.uk)

The copyright of individual parts of the supplement might differ from the CC BY 4.0 License. 
Table 1: Details of satellite imagery used in this study, including NDWI thresholds. Scenes used in subset area analysis are marked with a*.

\begin{tabular}{|c|c|c|c|c|c|c|c|}
\hline Scene ID & $\begin{array}{l}\text { Subset } \\
\text { analysis }\end{array}$ & UTM Zone & Date & Satellite/sensor & $\begin{array}{l}\text { Cloud } \\
\text { cover }(\%)\end{array}$ & $\begin{array}{l}\text { Data cover } \\
(\%)\end{array}$ & $\begin{array}{l}\text { NDWI } \\
\text { Threshold }\end{array}$ \\
\hline $\begin{array}{l}\text { S2B_MSIL1C_20200101T021549_N } \\
\text { 0208_R117_T47DNG }\end{array}$ & $*$ & $47 \mathrm{~S}$ & $01 / 01 / 2020$ & Sentinel 2 MSI & 54.4 & 45.6 & 0.25 \\
\hline $\begin{array}{l}\text { S2B_MSIL1C_20200111T021549_N } \\
\text { 0208_R117_T47DNG }\end{array}$ & $*$ & $47 \mathrm{~S}$ & $11 / 01 / 2020$ & Sentinel 2 MSI & 36 & 64 & 0.25 \\
\hline $\begin{array}{l}\text { LC08_L1GT_110107_20200113_202 } \\
00127\end{array}$ & & $47 \mathrm{~S}$ & $13 / 01 / 2020$ & Landsat 8 OLI & 0.02 & 99.8 & 0.25 \\
\hline $\begin{array}{l}\text { LC08_L1GT_113106_20200118_202 } \\
00128\end{array}$ & & $47 \mathrm{~S}$ & $18 / 01 / 2020$ & Landsat 8 OLI & 26.1 & 73.9 & 0.25 \\
\hline $\begin{array}{l}\text { LC08_L1GT_112107_20200127_202 } \\
00210\end{array}$ & & $47 \mathrm{~S}$ & $27 / 01 / 2020$ & Landsat 8 OLI & 2.8 & 97.2 & 0.25 \\
\hline $\begin{array}{l}\text { S2B_MSIL1C_20200131T021549_N } \\
\text { 0208_R117_T47DNG }\end{array}$ & $*$ & $47 \mathrm{~S}$ & $31 / 01 / 2020$ & Sentinel 2 MSI & 16.3 & 83.7 & 0.25 \\
\hline $\begin{array}{l}\text { LC08_L1GT_112107_20200228_202 } \\
00228\end{array}$ & $*$ & $47 \mathrm{~S}$ & $28 / 02 / 2020$ & Landsat 8 OLI & 0.06 & 99.9 & 0.25 \\
\hline $\begin{array}{l}\text { LC08_L1GT_111106_20191101_201 } \\
91114\end{array}$ & $*$ & $47 \mathrm{~S}$ & $11 / 01 / 2019$ & Landsat 8 OLI & 20.2 & 79.8 & $\begin{array}{ll}\text { No lakes } \\
\text { present }\end{array}$ \\
\hline $\begin{array}{l}\text { LC08_L1GT_111107_20191203_201 } \\
91216\end{array}$ & $*$ & $47 \mathrm{~S}$ & $03 / 12 / 2019$ & Landsat 8 OLI & 0 & 100 & $\begin{array}{ll}\text { No lakes } \\
\text { present }\end{array}$ \\
\hline $\begin{array}{l}\text { LC08_L1GT_111107_20191219_201 } \\
91226\end{array}$ & $*$ & $47 \mathrm{~S}$ & $19 / 12 / 2019$ & Landsat 8 OLI & $17.4 \%$ & $82.6 \%$ & 0.25 \\
\hline $\begin{array}{l}\text { S2B_MSIL1C_20190105T024559_N } \\
\text { 0207_R103_T47DMG }\end{array}$ & & $47 \mathrm{~S}$ & 05/01/2019 & Sentinel 2 MSI & $27.6 \%$ & $72.4 \%$ & 0.25 \\
\hline $\begin{array}{l}\text { S2B_MSIL1C_20190129T022559_N } \\
\text { 0207_R017_T47DPG }\end{array}$ & & $47 \mathrm{~S}$ & $29 / 01 / 2019$ & Sentinel 2 MSI & $20 \%$ & $80 \%$ & 0.25 \\
\hline $\begin{array}{l}\text { S2B_MSIL1C_20190129T022559_N } \\
\text { 0207_R017_T47DNG }\end{array}$ & $*$ & $47 \mathrm{~S}$ & $29 / 01 / 2019$ & Sentinel 2 MSI & $20 \%$ & $80 \%$ & 0.25 \\
\hline $\begin{array}{l}\text { LC08_L1GT_111107_20190218_201 } \\
90218\end{array}$ & $*$ & $47 \mathrm{~S}$ & $18 / 02 / 2019$ & Landsat 8 OLI & $0 \%$ & $100 \%$ & 0.25 \\
\hline $\begin{array}{l}\text { S2B_MSIL1C_20190228T022549_N } \\
\text { 0207_R017_T47DNG }\end{array}$ & $*$ & $47 \mathrm{~S}$ & $28 / 02 / 2019$ & Sentinel 2 MSI & $16.8 \%$ & $83.2 \%$ & 0.25 \\
\hline $\begin{array}{l}\text { S2A_MSIL1C_T47DNG_A013456_2 } \\
\text { 0180119T022550 }\end{array}$ & $*$ & $47 \mathrm{~S}$ & 19/01/2018 & Sentinel 2 MSI & $10.9 \%$ & $89.1 \%$ & 0.25 \\
\hline $\begin{array}{l}\text { S2A_MSIL1C_20180126T021551_N } \\
\text { 0206_R117_T47DNG }\end{array}$ & $*$ & $47 \mathrm{~S}$ & $26 / 01 / 2018$ & Sentinel 2 MSI & $0.2 \%$ & $99.8 \%$ & 0.25 \\
\hline $\begin{array}{l}\text { LC08_L1GT_113106_20180128_201 } \\
80207\end{array}$ & & $47 \mathrm{~S}$ & $28 / 01 / 2018$ & Landsat 8 OLI & $1.9 \%$ & $98.1 \%$ & 0.21 \\
\hline $\begin{array}{l}\text { S2A_L1C_T47DNG_A013742_2018 } \\
\text { 0208T022548 }\end{array}$ & $*$ & $47 \mathrm{~S}$ & $08 / 02 / 2018$ & Sentinel 2 MSI & $6.4 \%$ & $93.6 \%$ & 0.15 \\
\hline $\begin{array}{l}\text { LC08_L1GT_111107_20180215_201 } \\
80215\end{array}$ & $*$ & $47 \mathrm{~S}$ & $15 / 02 / 2018$ & Landsat 8 OLI & $0.07 \%$ & $99.9 \%$ & 0.20 \\
\hline $\begin{array}{l}\text { LC08_L1GT_111106_20170127_201 } \\
70214\end{array}$ & $*$ & $47 \mathrm{~S}$ & $27 / 01 / 2017$ & Landsat 8 OLI & $0.3 \%$ & $99.7 \%$ & 0.25 \\
\hline $\begin{array}{l}\text { S2A_MSIL1C_20170130T024551_N } \\
\text { 0204_R103_T47DMG }\end{array}$ & & $47 \mathrm{~S}$ & $30 / 01 / 2017$ & Sentinel 2 MSI & $15.5 \%$ & $84.5 \%$ & 0.25 \\
\hline
\end{tabular}




\begin{tabular}{|c|c|c|c|c|c|c|c|}
\hline $\begin{array}{l}\text { S2A_MSIL1C_T47DNG_A008408_2 } \\
\text { 0170131T021548 }\end{array}$ & * & $47 \mathrm{~S}$ & $31 / 01 / 2017$ & Sentinel 2 MSI & $1.7 \%$ & $98.3 \%$ & 0.25 \\
\hline $\begin{array}{l}\text { S2A_MSIL1C_20170131T021551_N } \\
\text { 0204_R117_T47DPG }\end{array}$ & & $47 \mathrm{~S}$ & $31 / 01 / 2017$ & Sentinel 2 MSI & 6.7 & 93.3 & 0.25 \\
\hline $\begin{array}{l}\text { S2A_MSIL1C_T47DPG_A008408_2 } \\
\text { 0170131T021548 }\end{array}$ & & $47 \mathrm{~S}$ & $31 / 01 / 2017$ & Sentinel 2 MSI & $6.6 \%$ & $93.4 \%$ & 0.25 \\
\hline $\begin{array}{l}\text { LC08_L1GT_111107_20171229_201 } \\
\text { 71229 }\end{array}$ & * & $47 \mathrm{~S}$ & 29/12/2017 & Landsat 8 OLI & $0 \%$ & $100 \%$ & 0.25 \\
\hline $\begin{array}{l}\text { LC08_L1GT_111107_20170228_201 } \\
\text { 70228 }\end{array}$ & & $47 \mathrm{~S}$ & $28 / 02 / 2017$ & Landsat 8 OLI & 9.5 & 95.5 & 0.25 \\
\hline $\begin{array}{l}\text { LC08_L1GT_111107_20160109_201 } \\
\text { 70405 }\end{array}$ & * & $47 \mathrm{~S}$ & 09/01/2016 & Landsat 8 OLI & $0.97 \%$ & $99.03 \%$ & 0.25 \\
\hline $\begin{array}{l}\text { LC08_L1GT_111107_20160125_201 } \\
\text { 70330 }\end{array}$ & * & $47 \mathrm{~S}$ & $25 / 01 / 2016$ & Landsat 8 OLI & $0.3 \%$ & $99.7 \%$ & 0.25 \\
\hline $\begin{array}{l}\text { LC08_L1GT_111106_20160226_201 } \\
\text { 70329 }\end{array}$ & * & $47 \mathrm{~S}$ & $26 / 02 / 2016$ & Landsat 8 OLI & $1.5 \%$ & $98.5 \%$ & 0.10 \\
\hline $\begin{array}{l}\text { LC08_L1GT_111107_20150106_201 } \\
\text { 80204_01_T2 }\end{array}$ & * & $47 \mathrm{~S}$ & $06 / 01 / 2015$ & Landsat 8 OLI & $5.7 \%$ & $94.3 \%$ & 0.25 \\
\hline $\begin{array}{l}\text { LC08_L1GT_111107_20150207_201 } \\
80204\end{array}$ & * & $47 \mathrm{~S}$ & $07 / 02 / 2015$ & Landsat 8 OLI & $17.9 \%$ & $82.1 \%$ & 0.25 \\
\hline $\begin{array}{l}\text { LC08_L1GT_111107_20150223_201 } \\
80204\end{array}$ & * & $47 \mathrm{~S}$ & $23 / 02 / 2015$ & Landsat 8 OLI & $13.1 \%$ & $86.9 \%$ & 0.25 \\
\hline $\begin{array}{l}\text { LC08_L1GT_111107_20140103_201 } \\
\text { 70427 }\end{array}$ & * & $47 \mathrm{~S}$ & $03 / 01 / 2014$ & Landsat 8 OLI & $27.2 \%$ & $72.8 \%$ & 0.25 \\
\hline $\begin{array}{l}\text { LC08_L1GT_111107_20140119_201 } \\
\text { 70426 }\end{array}$ & * & $47 \mathrm{~S}$ & $19 / 01 / 2014$ & Landsat 8 OLI & $14.3 \%$ & $85.7 \%$ & 0.25 \\
\hline $\begin{array}{l}\text { LE07_L1GT_112106_20130115_201 } \\
\text { 61126 }\end{array}$ & & $47 \mathrm{~S}$ & 15/01/2013 & Landsat 7 ETM+ & $24 \%$ & $76 \%$ & 0.25 \\
\hline $\begin{array}{l}\text { LC08_L1GT_111107_20131202_201 } \\
\text { 70428 }\end{array}$ & * & $47 \mathrm{~S}$ & $02 / 12 / 2013$ & Landsat 8 OLI & $2.9 \%$ & $97.1 \%$ & 0.25 \\
\hline $\begin{array}{l}\text { LE07_L1GT_111106_20120122_201 } \\
61203\end{array}$ & & $47 \mathrm{~S}$ & $22 / 01 / 2012$ & Landsat 7 ETM+ & $15 \%$ & $85 \%$ & 0.25 \\
\hline $\begin{array}{l}\text { LE07_L1GT_111106_20120223_201 } \\
61203\end{array}$ & * & $47 \mathrm{~S}$ & $23 / 02 / 2012$ & Landsat 7 ETM+ & $1 \%$ & $99 \%$ & 0.25 \\
\hline $\begin{array}{l}\text { LE07_L1GT_111107_20120223_201 } \\
\text { 61203 }\end{array}$ & $*$ & $47 \mathrm{~S}$ & $23 / 02 / 2012$ & Landsat 7 ETM+ & $0 \%$ & $100 \%$ & 0.25 \\
\hline $\begin{array}{l}\text { LE07_L1GT_111106_20110103_201 } \\
\text { 61210 }\end{array}$ & & $47 \mathrm{~S}$ & $03 / 01 / 2011$ & Landsat 7 ETM+ & $0 \%$ & $100 \%$ & 0.25 \\
\hline $\begin{array}{l}\text { LE07_L1GT_111107_20110204_201 } \\
\text { 61211 }\end{array}$ & * & $47 \mathrm{~S}$ & $04 / 02 / 2011$ & Landsat 7 ETM+ & $0 \%$ & $100 \%$ & 0.25 \\
\hline $\begin{array}{l}\text { LE07_L1GT_112107_20110227_201 } \\
61210\end{array}$ & & $47 \mathrm{~S}$ & $27 / 02 / 2011$ & Landsat 7 ETM+ & $2 \%$ & $98 \%$ & 0.15 \\
\hline $\begin{array}{l}\text { LE07_L1GT_111107_20111103_201 } \\
\text { 61205 }\end{array}$ & * & $47 \mathrm{~S}$ & $03 / 11 / 2011$ & Landsat 7 ETM+ & 0 & 100 & $\begin{array}{l}\text { No lakes } \\
\text { present }\end{array}$ \\
\hline $\begin{array}{l}\text { LE07_L1GT_111107_20111119_201 } \\
\text { 61205 }\end{array}$ & $*$ & $47 \mathrm{~S}$ & $19 / 11 / 2011$ & Landsat 7 ETM+ & 0 & 100 & $\begin{array}{ll}\text { No lakes } \\
\text { present }\end{array}$ \\
\hline $\begin{array}{l}\text { LE07_L1GT_111107_20111205_201 } \\
61204\end{array}$ & $*$ & $47 \mathrm{~S}$ & $5 / 12 / 2011$ & Landsat 7 ETM+ & 0 & 100 & $\begin{array}{ll}\text { No lakes } \\
\text { present }\end{array}$ \\
\hline $\begin{array}{l}\text { LE07_L1GT_110107_20100210_201 } \\
61217\end{array}$ & & $47 \mathrm{~S}$ & $10 / 02 / 2010$ & Landsat 7 ETM+ & $0 \%$ & $100 \%$ & 0.25 \\
\hline $\begin{array}{l}\text { LE07_L1GT_113106_20100215_201 } \\
\text { 61216 }\end{array}$ & $*$ & $47 \mathrm{~S}$ & $15 / 02 / 2010$ & Landsat 7 ETM+ & $2 \%$ & $98 \%$ & 0.25 \\
\hline
\end{tabular}




\begin{tabular}{|c|c|c|c|c|c|c|c|}
\hline $\begin{array}{l}\text { LE07_L1GT_113107_20100215_201 } \\
61216\end{array}$ & * & $47 \mathrm{~S}$ & $15 / 02 / 2010$ & Landsat 7 ETM+ & $1 \%$ & $99 \%$ & 0.15 \\
\hline $\begin{array}{l}\text { LE07_L1GT_111107_20100217_201 } \\
61215\end{array}$ & * & $47 \mathrm{~S}$ & $17 / 02 / 2010$ & Landsat 7 ETM+ & $0 \%$ & $100 \%$ & 0.25 \\
\hline $\begin{array}{l}\text { LE07_L1GT_112106_20100224_201 } \\
61217\end{array}$ & * & $47 \mathrm{~S}$ & $24 / 02 / 2010$ & Landsat 7 ETM+ & $0 \%$ & $100 \%$ & 0.20 \\
\hline $\begin{array}{l}\text { LE07_L1GT_112106_20101107_201 } \\
\text { 61212 }\end{array}$ & * & $47 \mathrm{~S}$ & $07 / 11 / 2010$ & Landsat 7 ETM+ & $0 \%$ & $100 \%$ & 0.25 \\
\hline $\begin{array}{l}\text { LE07_L1GT_114107_20090102_201 } \\
\text { 61223 }\end{array}$ & & $47 \mathrm{~S}$ & $02 / 01 / 2009$ & Landsat 7 ETM+ & $1 \%$ & $99 \%$ & 0.25 \\
\hline $\begin{array}{l}\text { LE07_L1GT_111106_20091231_201 } \\
\text { 61216 }\end{array}$ & & $47 \mathrm{~S}$ & $31 / 12 / 2009$ & Landsat 7 ETM+ & $13 \%$ & $87 \%$ & 0.20 \\
\hline $\begin{array}{l}\text { LE07_L1GT_113107_20080109_201 } \\
\text { 61231 }\end{array}$ & & $47 \mathrm{~S}$ & 09/01/2008 & Landsat 7 ETM+ & $10 \%$ & $90 \%$ & 0.25 \\
\hline $\begin{array}{l}\text { LE07_L1GT_111107_20080127_201 } \\
61230\end{array}$ & & $47 \mathrm{~S}$ & $27 / 01 / 2008$ & Landsat 7 ETM+ & $0 \%$ & $100 \%$ & 0.25 \\
\hline $\begin{array}{l}\text { LE07_L1GT_111106_20080127_201 } \\
61230\end{array}$ & & $47 \mathrm{~S}$ & $27 / 01 / 2008$ & Landsat 7 ETM+ & $0 \%$ & $100 \%$ & 0.25 \\
\hline $\begin{array}{l}\text { LE07_L1GT_111107_20081126_201 } \\
61223\end{array}$ & * & $47 \mathrm{~S}$ & $26 / 11 / 2008$ & Landsat 7 ETM+ & 1 & 99 & $\begin{array}{l}\text { No lakes } \\
\text { present }\end{array}$ \\
\hline $\begin{array}{l}\text { LE07_L1GT_111107_20081212_201 } \\
61223\end{array}$ & * & $47 \mathrm{~S}$ & $12 / 12 / 2008$ & Landsat 7 ETM+ & 0 & 100 & $\begin{array}{l}\text { No lakes } \\
\text { present }\end{array}$ \\
\hline $\begin{array}{l}\text { LE07_L1GT_112107_20070115_201 } \\
\text { 70105 }\end{array}$ & & $47 \mathrm{~S}$ & $15 / 01 / 2007$ & Landsat 7 ETM+ & $0 \%$ & $100 \%$ & 0.25 \\
\hline $\begin{array}{l}\text { LE07_L1GT_113107_20070122_201 } \\
\text { 70105 }\end{array}$ & * & $47 \mathrm{~S}$ & $22 / 01 / 2007$ & Landsat 7 ETM+ & $0 \%$ & $100 \%$ & 0.25 \\
\hline $\begin{array}{l}\text { LE07_L1GT_113107_20070223_201 } \\
\text { 70105 }\end{array}$ & & $47 \mathrm{~S}$ & 23/02/2007 & Landsat 7 ETM+ & $21 \%$ & $79 \%$ & 0.25 \\
\hline $\begin{array}{l}\text { LE07_L1GT_112106_20061230_201 } \\
\text { 70106 }\end{array}$ & & $47 \mathrm{~S}$ & $30 / 12 / 2006$ & Landsat 7 ETM+ & $2 \%$ & $98 \%$ & 0.27 \\
\hline $\begin{array}{l}\text { LE07_L1GT_112106_20040208_201 } \\
\text { 70123 }\end{array}$ & & $47 \mathrm{~S}$ & $08 / 02 / 2004$ & Landsat 7 ETM+ & $19 \%$ & $81 \%$ & 0.25 \\
\hline $\begin{array}{l}\text { LE07_L1GT_112107_20040208_201 } \\
\text { 70123 }\end{array}$ & & $47 \mathrm{~S}$ & $08 / 02 / 2004$ & Landsat 7 ETM+ & $0 \%$ & $100 \%$ & 0.25 \\
\hline $\begin{array}{l}\text { LE07_L1GT_112106_20021219_201 } \\
\text { 70127 }\end{array}$ & & $47 \mathrm{~S}$ & $19 / 12 / 2002$ & Landsat 7 ETM+ & $0 \%$ & $100 \%$ & 0.20 \\
\hline $\begin{array}{l}\text { LE07_L1GT_112107_20021219_201 } \\
\text { 70127 }\end{array}$ & & $47 \mathrm{~S}$ & $19 / 12 / 2002$ & Landsat 7 ETM+ & $2 \%$ & $98 \%$ & 0.25 \\
\hline $\begin{array}{l}\text { LE07_L1GT_111106_20000206_201 } \\
\text { 70213 }\end{array}$ & * & $47 \mathrm{~S}$ & $06 / 02 / 2000$ & Landsat 7 ETM+ & $8 \%$ & $92 \%$ & 0.25 \\
\hline $\begin{array}{l}\text { LE07_L1GT_111107_20000206_201 } \\
\text { 70213 }\end{array}$ & * & $47 \mathrm{~S}$ & $06 / 02 / 2000$ & Landsat 7 ETM+ & $0 \%$ & $100 \%$ & 0.25 \\
\hline $\begin{array}{l}\text { LT05_L1GS_114107_19910210_201 } \\
\text { 70127 }\end{array}$ & & $47 \mathrm{~S}$ & $10 / 02 / 1991$ & Landsat $5 \mathrm{TM}$ & $0 \%$ & $100 \%$ & 0.25 \\
\hline $\begin{array}{l}\text { LT05_L1GS_114106_19910210_201 } \\
\text { 70127 }\end{array}$ & & $47 \mathrm{~S}$ & $10 / 02 / 1991$ & Landsat 5 TM & $0 \%$ & $100 \%$ & 0.25 \\
\hline $\begin{array}{l}\text { LT05_L1GS_112107_19910212_201 } \\
\text { 70127 }\end{array}$ & & $47 \mathrm{~S}$ & 12/02/1991 & Landsat $5 \mathrm{TM}$ & $1 \%$ & $99 \%$ & 0.25 \\
\hline $\begin{array}{l}\text { LT05_L1GS_112107_19910228_201 } \\
\text { 70127 }\end{array}$ & & $47 \mathrm{~S}$ & 28/02/1991 & Landsat $5 \mathrm{TM}$ & $11 \%$ & $89 \%$ & 0.25 \\
\hline $\begin{array}{l}\text { LT04_L1GS_112106_19891113_201 } \\
\text { 70201 }\end{array}$ & & $47 \mathrm{~S}$ & $13 / 11 / 1989$ & Landsat 4 TM & $15 \%$ & $85 \%$ & 0.25 \\
\hline
\end{tabular}




\begin{tabular}{|l|l|l|l|l|l|l|l|}
\hline $\begin{array}{l}\text { LT04_L1GS_112107_19891113_201 } \\
\text { 70201 }\end{array}$ & & $47 \mathrm{~S}$ & $13 / 11 / 1989$ & Landsat 4 TM & $0 \%$ & $100 \%$ & 0.25 \\
\hline $\begin{array}{l}\text { LM01_L1GS_119107_19740223_201 } \\
\text { 80426 }\end{array}$ & & $47 \mathrm{~S}$ & $23 / 02 / 1974$ & Landsat 1 MSS & $6 \%$ & $94 \%$ & 0.15 \\
\hline
\end{tabular}

10

15 

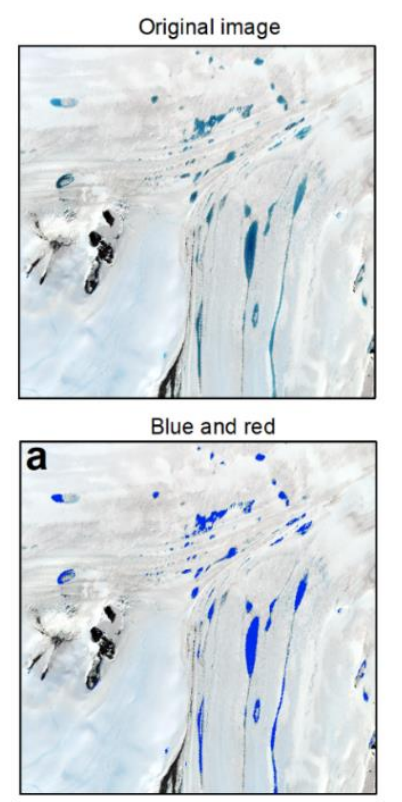

Green and near-infrared

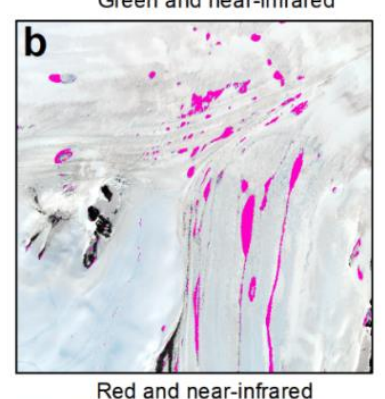

Red and near-infrared

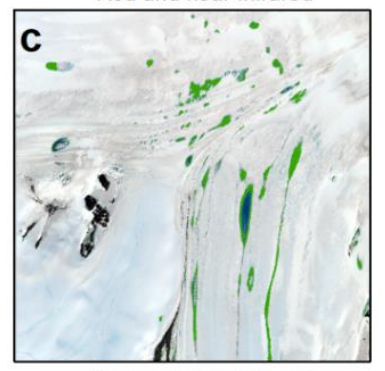

Blue and near-infrared

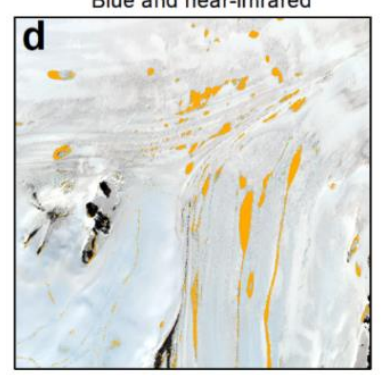

Original image

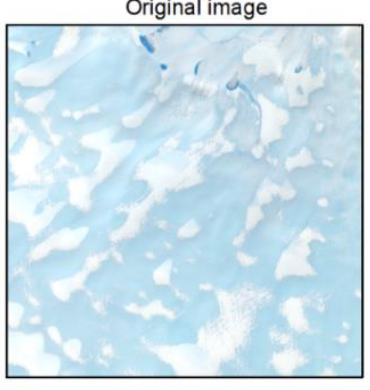

Blue and red

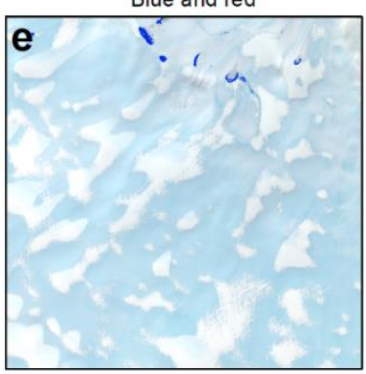

Green and near-infrared

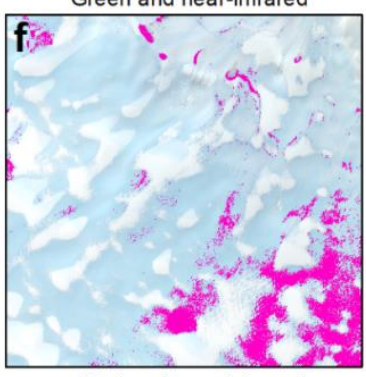

Red and near-infrared

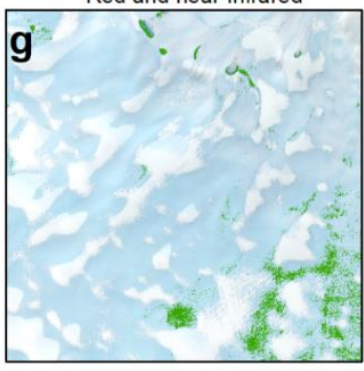

Blue and near-infrared

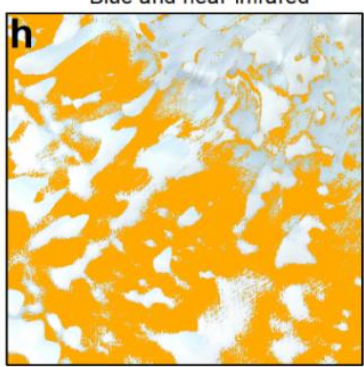

Supplementary Figure 1. Difference between different NDWI methods over an area snow-covered ice on which supraglacial lakes have formed (a - d) and exposed blue ice (e - h). The low near-infrared spectral reflectivity of snow-free blue ice results in pixels in blue ice areas being falsely classified as being water-covered. Background image is a Sentinel 2A image captured on 26th January 2018. 

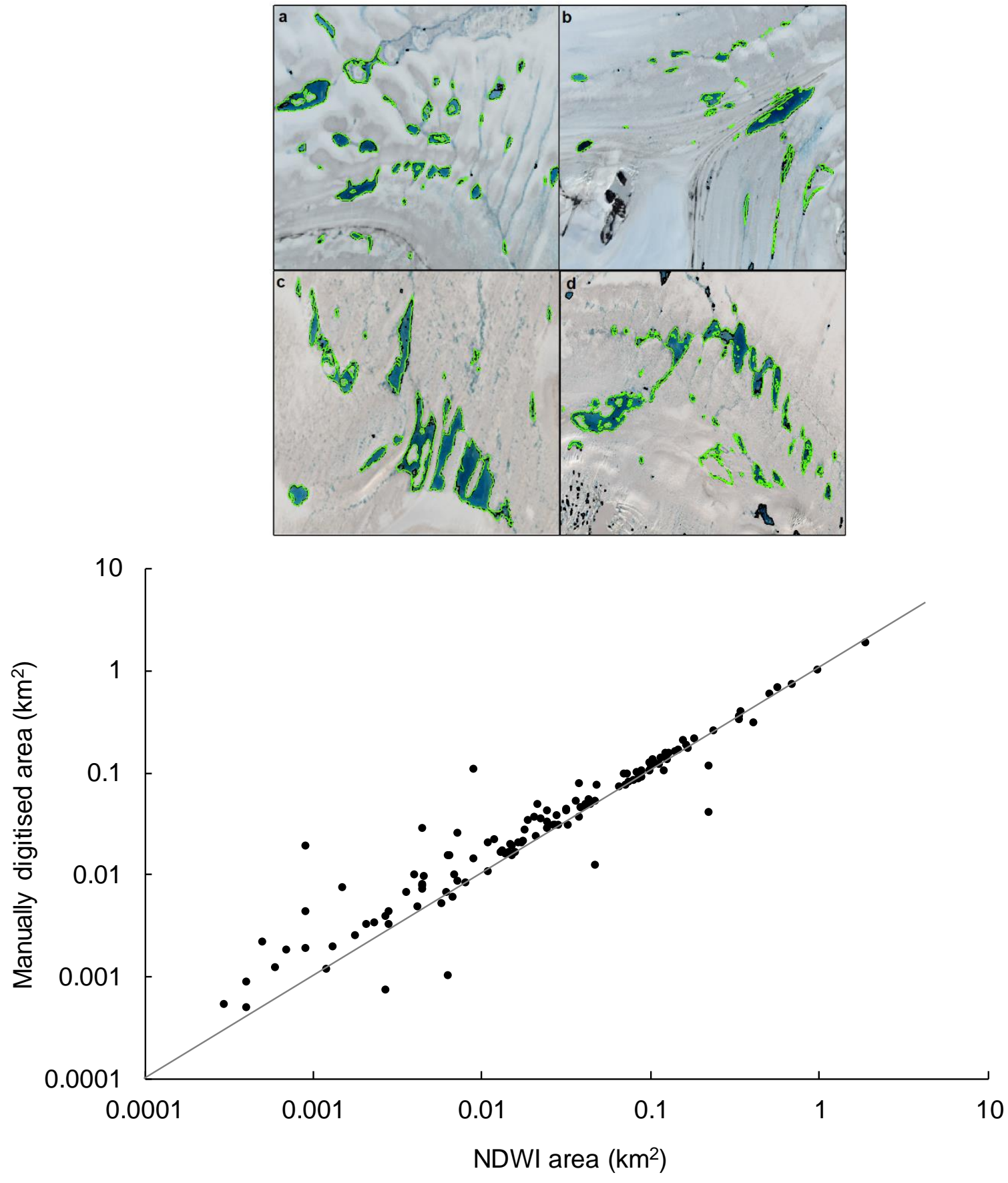

Supplementary Figure 2: Scatterplot of lake outlines comparing those derived from the Normalised Difference Water Index (black) to those derived from manual delineation (green) in four sample areas (a-d). Note the strong correlation of the linear regression (R2 $=0.979)$, but with a higher scatter at very low lake areas. Lakes were manually delineated from a Landsat 8 scene $(03 / 01 / 2014)$ in a$80 \mathrm{~b}$ and from a Sentinel 2 scene $(\mathbf{1 9 / 0 1 / 2 0 1 8 )}$ in c-d. 


\section{Assessing the effect of sensor resolution on lake detection and areas}

In order to assess the impact of sensor resolution on lake detection and lake extents, we extracted lake areas from the overlapping portion of a Sentinel 2B and Landsat 8 scene taken on $11^{\text {th }}$ January 2020, using the NDWI ( 0.25 threshold) and a minimum size threshold of two pixels (see Section 3.2 of main paper). We detected larger numbers of smaller lakes from

85 Sentinel 2B imagery (minimum of $200 \mathrm{~m}^{2}$ ) compared to Landsat 8 (minimum of $450 \mathrm{~m}^{2}$ ) (Supplementary Figure 3), but there is generally a very good agreement between lake areas derived from the two sensors ( $22=0.9923, \mathrm{RMSE}=0.0003$ ). Lake areas derived from Sentinel 2 are generally higher, which we attribute to pixels along the outer edges of lakes being more accurately classified as water.
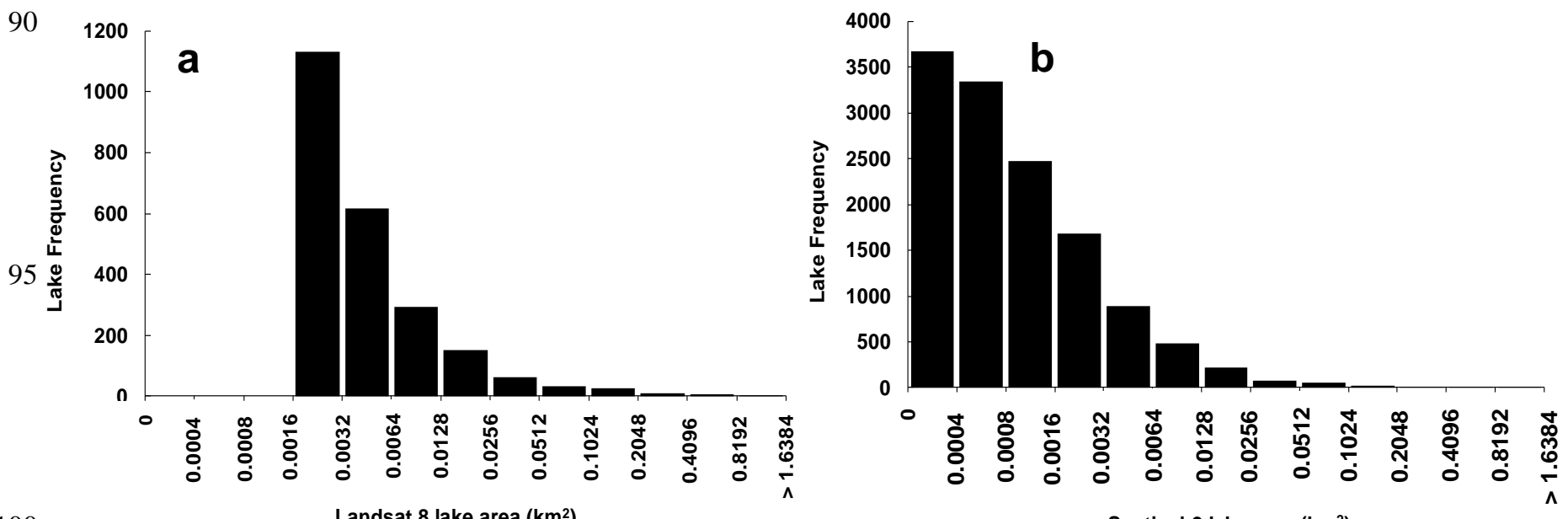

100

Landsat 8 lake area $\left(\mathrm{km}^{2}\right)$

Sentinel 2 lake area $\left(\mathrm{km}^{2}\right)$

105

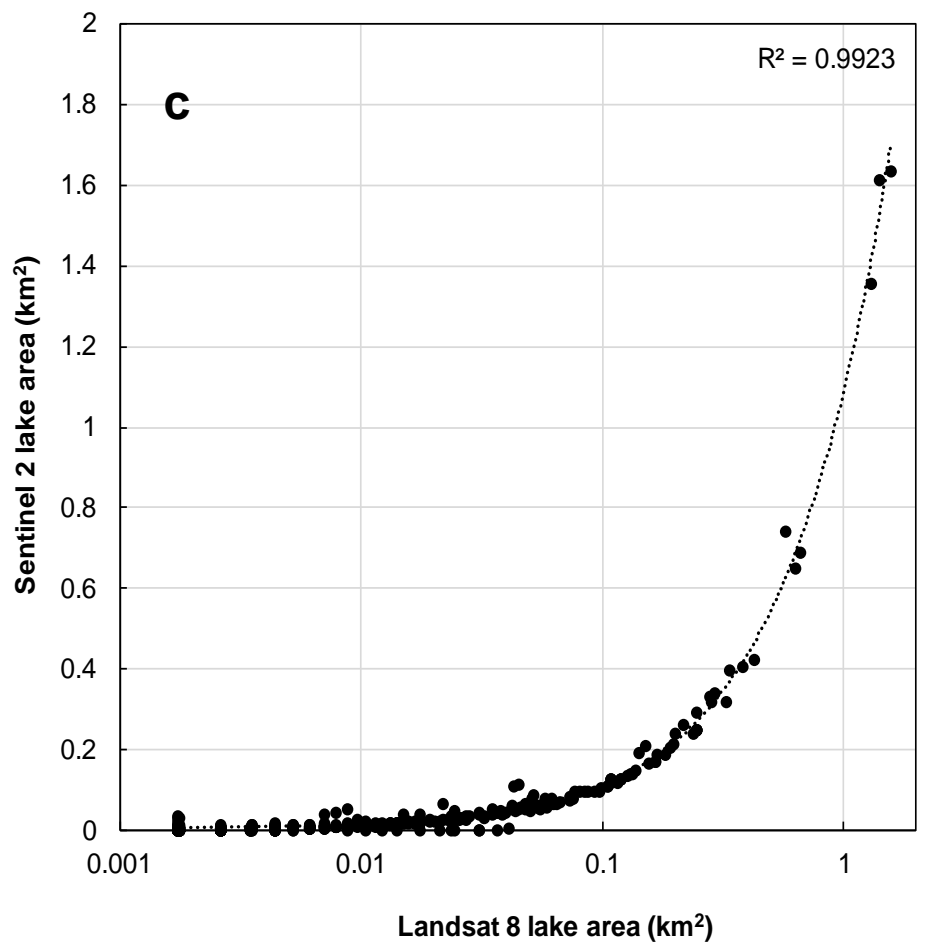


Supplementary Figure 3. Lake area-frequency distribution from a Landsat 8 scene on $11^{\text {th }}$ January 2020 (a). Lake area-frequency
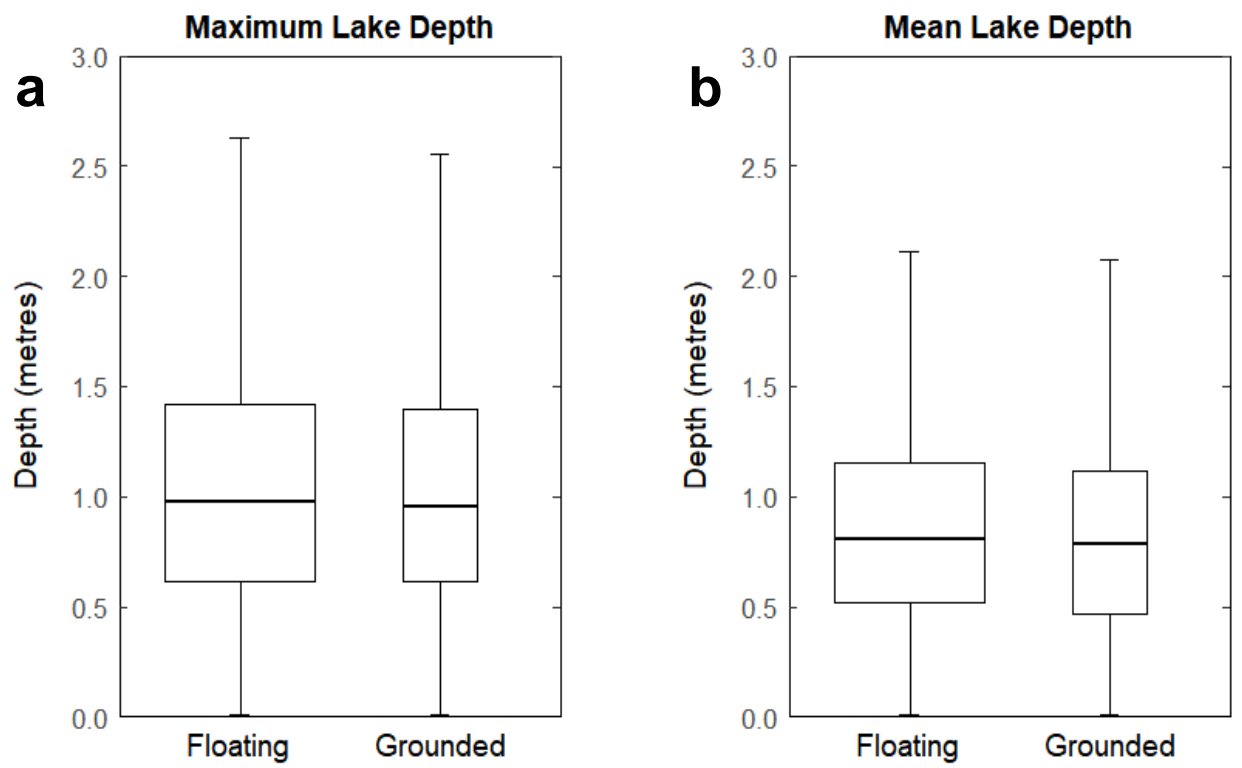

130
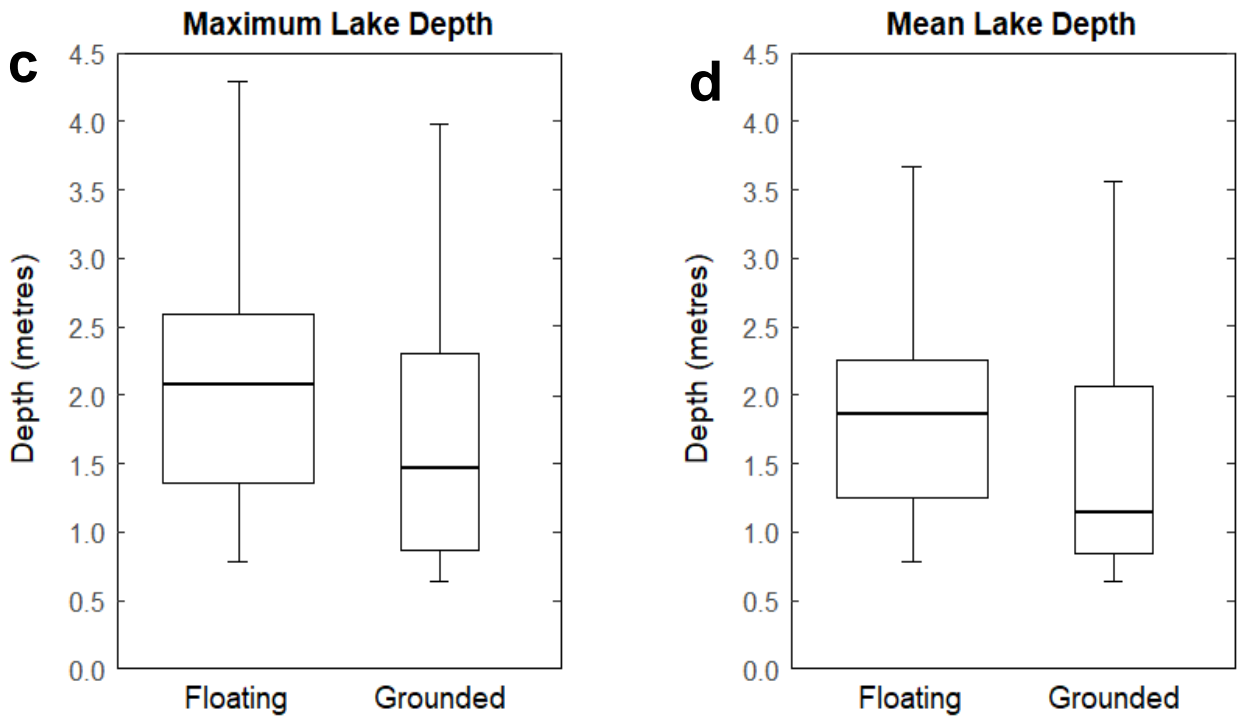

Supplementary Figure 4. Box plots of maximum depth and mean depth of SGLs on Shackleton Ice Shelf and on grounded ice within

145 the sub-region (Figure 1, green box) from 2000 to $2020(a, b)$ and from the date when the deepest lakes formed, 03/01/2014 (c, d). On each box, the bold line marks the median and the edges of the box are the $25^{\text {th }}$ and $75^{\text {th }}$ percentiles (q1 and q3). The length of the upper and lower whiskers is $\mathrm{q} 3+1.5 *(\mathrm{q} 3-\mathrm{q} 1)$ and $\mathrm{q} 1-1.5 *(\mathrm{q} 3-\mathrm{q} 1)$ respectively. Box widths are proportional to the number of lakes recorded on floating and grounded ice. 

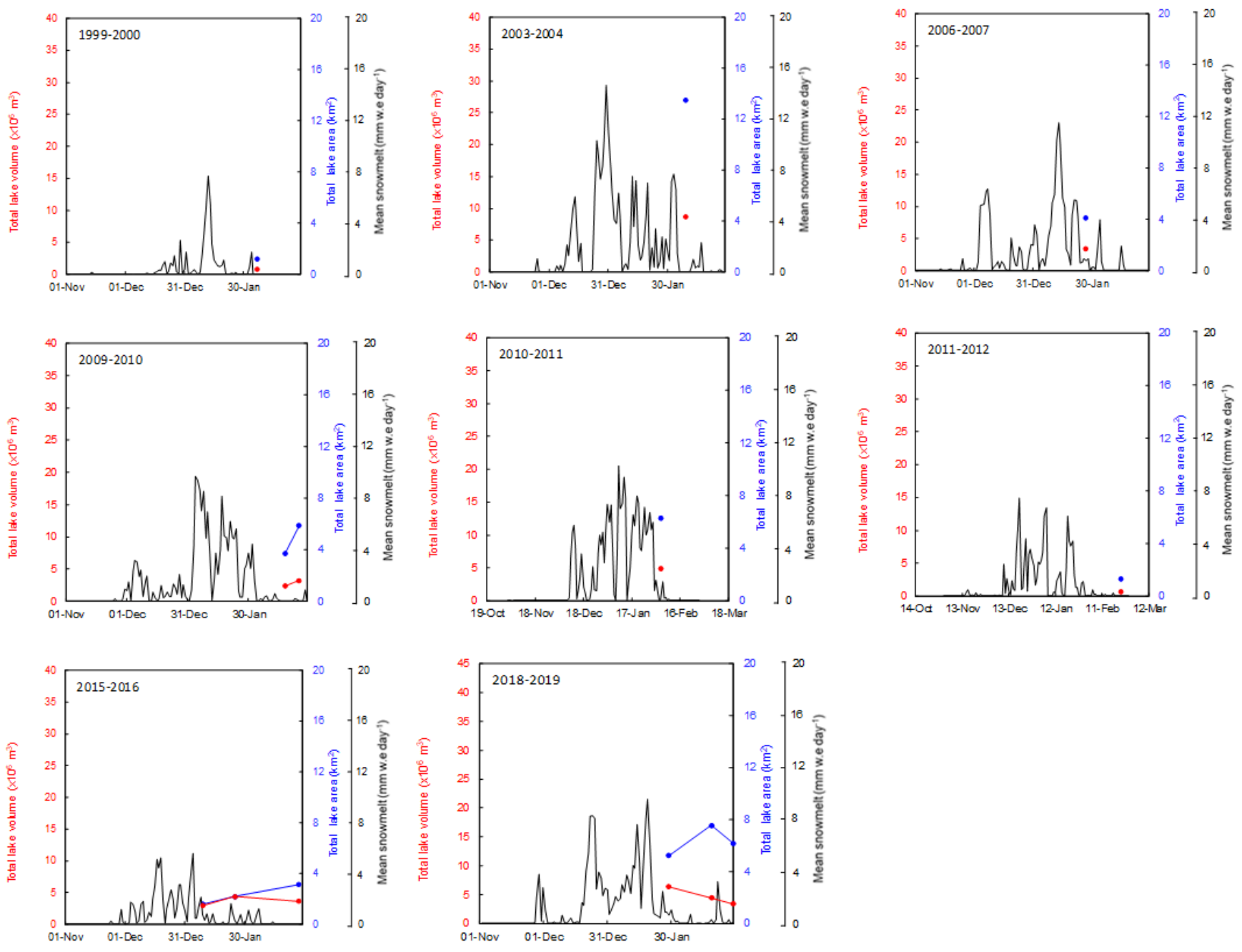

Supplementary Figure 5. Seasonal variations in total lake area, volume and modelled snowmelt for melt seasons with insufficient imagery to be able to determine whether peak area and volume follow a spike in surface melt. Snowmelt rates are mean values over the entire ice shelf. 


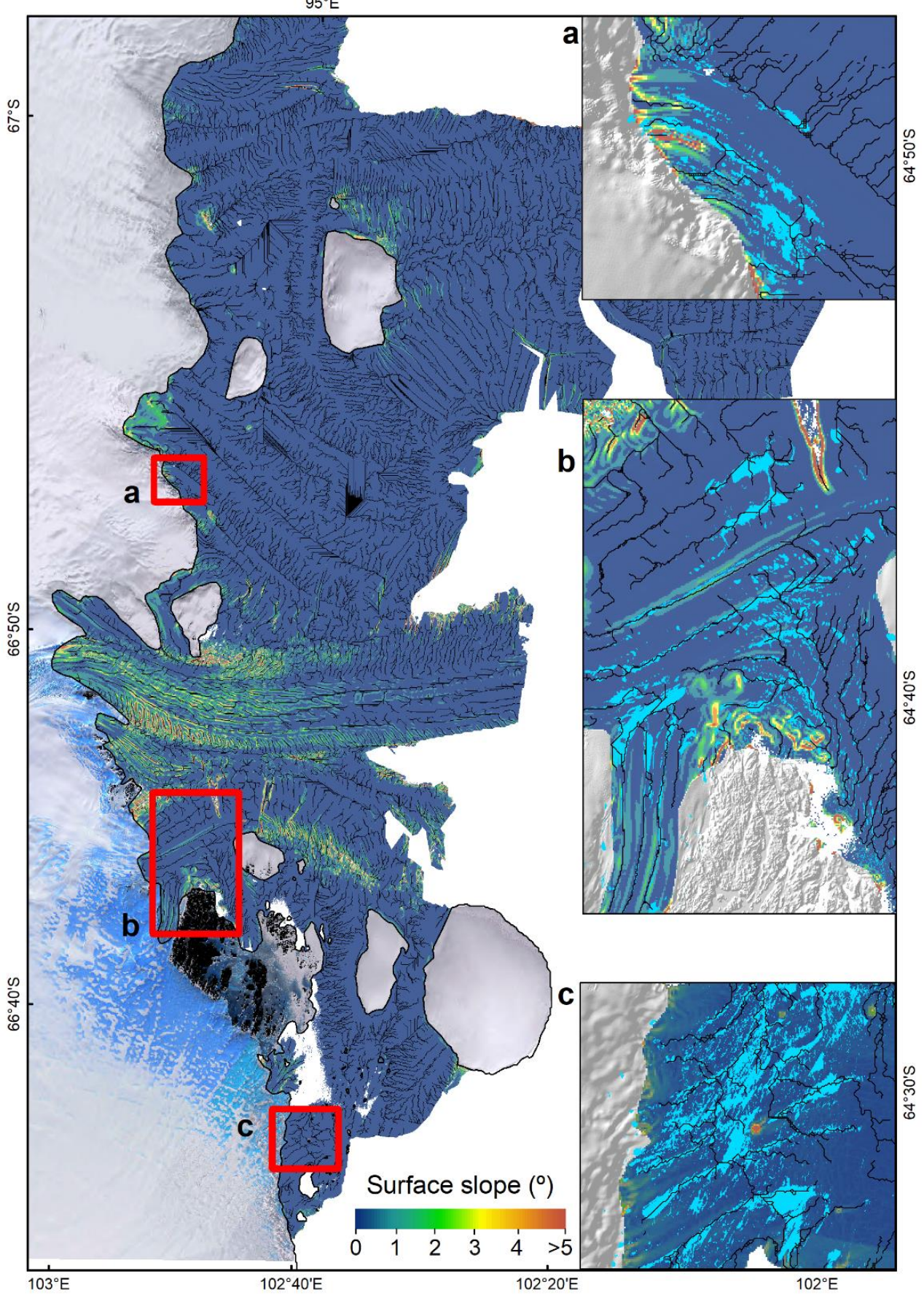

Supplementary Figure 6. Predicted surface meltwater routing on Shackleton Ice Shelf (black channels) overlain by ice shelf surface slopes and by SGLs mapped in this study (light blue), assuming widespread firn saturation across the ice shelf. Surface meltwater is predicted to be fed from higher slope areas upstream and converge in topographical lows, where it will be exported off the ice shelf along troughs. Insets show SGLs mapped in this study closely correspond with predicted paths of meltwater routing. 